\title{
A model of knowledge management system in managing knowledge of software testing environment
}

\begin{abstract}
Software testing is the process on how to identify and deliver the software as a product based on the specification that has been given and required by the users. In order to ensure that the product is working based on the user specification, there are many people who are working together for that purposes as a community of practice $(\mathrm{CoP})$. The $\mathrm{CoP}$ in software testing environment is including the system designer, programmer, and system tester as well as the user by himself. Based on this scenario of working together or collaboratively in order to avoid a lot of mistake or errors and causes the software failure, which may be found during the processes of software testing process, so that there is a need for CoP to have a tool called knowledge management system (KMS) in managing the knowledge of best practice and lesson learnt. The paper will discuss the concept on how the KMS is offering of its processes through knowledge life cycle which starting from knowledge acquisition, knowledge storing, knowledge dissemination, and knowledge application. Therefore, by using the model of KMS in managing knowledge of software testing, CoP can utilize the knowledge in KMS and it will reduces the mistake or errors, so that they can delivered a good product besides to enhance the quality of software of the particular users.
\end{abstract}

Keyword: Software testing; Collaboration; Knowledge management; Knowledge life cycle; Software quality 